\title{
Enhancing the information of nonlinear SU $(1,1)$ quantum systems interacting with a two-level atom
}

\section{M.Y. Abd-Rabbou ( $\nabla$ m.elmalky@azhar.edu.eg)}

Al-Azhar University Faculty of Science https://orcid.org/0000-0003-3197-4724

S. I. Ali

Al-Azhar University Faculty of Science

M. M. A. Ahmed

Al-Azhar University Faculty of Science

\section{Research Article}

Keywords:

Posted Date: March 3rd, 2022

DOI: https://doi.org/10.21203/rs.3.rs-1281051/v1

License: (c) (1) This work is licensed under a Creative Commons Attribution 4.0 International License.

Read Full License 


\title{
Enhancing the information of nonlinear $S U(1,1)$ quantum systems interacting with a two-level atom
}

\author{
M. Y. Abd-Rabbou 1, S. I. Ali and M. M. A. Ahmed \\ Mathematics Department, Faculty of Science, Al-Azhar University, Nasr City 11884, Cairo, Egypt.
}

\begin{abstract}
The effect of nonlinearity, initial atomic state, and different resonance cases on the interaction between nonlinear $\mathrm{SU}(1,1)$ quantum states and a two-level atom is discussed. The optimal behaviours of decoherence, entanglement and quantum coherence are predicted via using the skew information, tomographic entropy, and the relative entropy of coherence, respectively. It is shown that the detuning parameter has a destructive effect on the coherence and consequently on the entanglement if the quantum system is regulated in the ideal $\mathrm{SU}(1,1)$ quantum systems. For the nonlinear SU $(1,1)$ quantum systems, the ability to suppress the decay of entanglement induced by the detuning may be increased by preparing the initial atomic state in its excited state.
\end{abstract}

\section{Introduction:}

The control of entanglement for a quantum system has attracted the interest of many physicists for its importance in the processing of quantum information, as it is the foundation of quantum computation and communication [1-3]. Additionally, many theoretical and experimental schemes have been proposed for enhancing the entanglement and quantum correlation [4 7]. The measuring of entanglement degree has been obtained via different methods, such as von Neumann entropy [8,9], concurrence [10], negativity [11, 12], and the entanglement of formation [13]. Likewise, the entanglement path has also been predicted by some measurements, such as entropy squeezing [14], tomographic entropy [15, 16], Wigner function [17, and quantum uncertainty and local quantum Fisher information [18.

As is well known, there are interesting issues concerning the light-matter interaction in quantum optics. These issues are the atom-field interaction [19 21], atom-atom interaction [22, 23], and field-field interaction [24,25]. These interactions contained many natural phenomena that have been observed in the experimental systems. Moreover, these types of interactions may be described by some mathematical tools to switch from one structure to another. The interaction between a set of two-level atoms and a quantized field has been transformed into three modes of electromagnetic fields [26], atom-atom or field-atom-interactions [27, 28]. In this context, we aim to investigate the interaction between a twolevel atom and the category of the $\mathrm{SU}(1,1)$ Lie algebra, where the atom may be considered as a particle regularized in the $\mathrm{SU}(2)$ Lie algebra. The interaction between $\mathrm{SU}(1,1)$ and $\mathrm{SU}(2)$ quantum systems have been studied by many authors [14,29]. The effect of damping reservoir for even case of Barut-Girardello states at $k=1 / 4$ have been discussed [30]. The influence of coupling parameters of the external classical field system on the $\mathrm{SU}(1,1)$ and $\mathrm{SU}(2)$ interaction have been examined 31, 32. The relation between quantum Fisher information (QFI) 33, 34 and quantum entanglement of two atoms that interact with two non-degenerate modes has been investigated [35]. The interaction between $\mathrm{SU}(1,1)$ Lie algebra and three-level atom in presence of laser field which related to ideal and real laser is presented [32]. Via the spherical harmonics, Barut-Girardello states can be generated, which describe the system entanglement [36]. Superposition of Perelomov leading to SU $(1,1)$ coherent states of the Gilmore-Perelomov type has been proposed by using a Jaynes-Cummings model with intensity dependent coupling and an external field [37.

\footnotetext{
${ }^{1}$ E-mail:m.elmalky@azhar.edu.eg
} 
Our motivation is to examine the temporal evolution of three different types of quantum information; skew information, tomographic entropy, and relative entropy of coherent. In which we address the subject of what is the correlation between the three quantifying measurements and subsystems entanglement control? The main task is to study the influence of initial setting angles, the detuning parameter, and the non-linear term on the relation between the three quantities, where there is a strong correlation for ideal systems [38 40$]$. So, is different parameters breaking these correlations?

The paper is arranged as follows: In section 2, we introduce the physical discretion of the Hamiltonian operator for the non-linear system. As well, we obtained the temporal evolution of the wave function via using Heisenberg equations of motions. The mathematical definitions of the skew information, tomographic entropy and relative entropy of coherent entropy are proposed in section 3. Section 4 is devoted to displaying the discussion and numerical results.

\section{Model and solution}

We shall consider the Hamiltonian model consists of a two-level atom interacting with $S U(1,1)$ quantum systems, which takes the following form. $(\hbar=1)$,

$$
\hat{H}=\omega k_{z}+\sum_{i=1}^{2} \Omega_{i} S_{i i}+\lambda\left(S_{12} \hat{R}+\hat{R}^{\dagger} S_{21}\right)
$$

where $\omega$ and $\Omega_{j}, j=1,2$ are the radiation $S U(1,1)$ frequency and the atomic transition, respectively, while $\lambda_{j}$ is the coupling constant between the non linear $S(1,1)$ quantum system and the atomic state. The operators $S_{i j}=|i\rangle\langle j|$ are the transition operators for the atomic states, with $\left[S_{i j}, S_{k l}\right]=S_{i l} \delta_{k j}-S_{k j} \delta_{i l}$. Likewise, the operators $\hat{R}=K_{+} f\left(K_{z}\right)$ and $\hat{R}^{\dagger}=K_{+} f\left(K_{Z}\right)$ are the non-linear operator in $S U(1,1)$ Lie algebra, with the generators $K_{z}$ and $K_{ \pm}$. These generators satisfy the following commutation relations;

$$
\left[\hat{K}_{z}, \hat{K}_{ \pm}\right]= \pm \hat{K}_{ \pm}, \quad\left[\hat{K}_{-}, \hat{K}_{+}\right]=2 \hat{K}_{z},\left[\hat{R}, \hat{K}_{z}\right]=-\hat{R}, \text { and }\left[\hat{R}^{\dagger}, \hat{K}_{z}\right]=\hat{R}^{\dagger}
$$

and the related Casmir operator $K$ with,

$$
K^{2}=K_{z}^{2}-\frac{1}{2}\left(K_{+} K_{-}+K_{-} K_{+}\right)
$$

These operators have the corresponding eigenvalues;

$$
\begin{aligned}
& K_{z}|m, k\rangle=(m+k)|m, k\rangle, \quad \hat{K}_{+}|m, k\rangle=\sqrt{((m+1)(m+2 k)}|m+1, k\rangle, \\
& \hat{K}_{-}|m, k\rangle=\sqrt{(m(m+2 k-1))}|m-1, k\rangle, \quad \hat{K}^{2}|m, k\rangle=k(k-1)|m, k\rangle,
\end{aligned}
$$

here $m$ represents any non-negative integer, and $k$ is the Bargmann index.

Since the $S U(1,1)$ Lie algebra can be expressed in terms of boson annihilation and creation operators, it is isomorphic to the Lie algebra of the non-compact $S U(1,1)$ group. Therefore for a single-mode operator $\hat{a}$, we define

$$
\hat{K}_{+}=\frac{1}{2} \hat{a}^{\dagger 2}, \quad \hat{K}_{-}=\frac{1}{2} \hat{a}^{2}, \quad \hat{K}_{z}=\frac{1}{2}\left(\hat{a}^{\dagger} \hat{a}+\frac{1}{2}\right) .
$$

Whereas for the two-mode bosonic representation they take the form

$$
\hat{K}_{+}=\hat{a}^{\dagger} \hat{b}^{\dagger}, \quad \hat{K}_{-}=\hat{a} \hat{b}, \quad K_{z}=\frac{1}{2}\left(\hat{a}^{\dagger} \hat{a}+\hat{b}^{\dagger} \hat{b}+1\right)
$$


Alternatively, in the Holstien-Primakoff representation 41,42 , the generators $\hat{K}_{ \pm}$and $\hat{K}_{z}$ are given by

$K_{+}=\sqrt{2 k-1+n} \hat{a}^{\dagger}, \quad K_{-}=\hat{a} \sqrt{2 k-1+n}$, with $\hat{K}_{z}=\hat{K}+\hat{n}$

This means that the interaction between the quantum system $\mathrm{SU}(1,1)$ and the atomic system $\mathrm{SU}(2)$ is very rich and would give us a wide scope for studying the nature of this kind of interaction. The Heisenberg equation of motion for any operator $\hat{O}(t)$ is given by $i \frac{d \hat{O}}{d t}=[\hat{O}, \hat{H}]$. Thus the equations of motion for $\hat{S}_{j j}$ and $\hat{K}_{z}$ are given by

$$
i \frac{d \hat{S}_{11}}{d t}=\lambda\left(\hat{R} \hat{S}_{12}-\hat{S}_{21} \hat{R}^{\dagger}\right), \quad i \frac{d \hat{S}_{22}}{d t}=-\lambda\left(\hat{R} \hat{S}_{12}-\hat{S}_{21} \hat{R}^{\dagger}\right), \quad i \frac{d \hat{K}_{z}}{d t}=-\lambda\left(\hat{R} S_{12}-S_{21} \hat{R}^{\dagger}\right)
$$

Therefore we have the following constant of motion $\hat{N}=\hat{K}_{z}+\frac{1}{2}\left(\hat{S}_{11}-\hat{S}_{22}\right)$. However, we can write the Hamiltonian of equation(1) as,

$$
\hat{H}=\omega \hat{N}+\hat{C}
$$

where $\hat{C}=\frac{\Delta}{2}\left(\hat{S}_{11}-\hat{S}_{22}\right)+\lambda\left(S_{12} \hat{R}+\hat{R}^{\dagger} S_{21}\right)$.

The time evolution operator $U(t)=e^{-i \hat{H} t}=e^{-i \hat{N} t} e^{-i \hat{C} t}$, which can be written by employ the method of Stenholm [43 45] working in the atomic basis and in the interaction picture. After straightforward calculations, we can write the time evolution operator as:

$$
\hat{U}(t)=e^{-i \hat{N} t} e^{-i \hat{C} t}=e^{-i \hat{N} t}\left[\begin{array}{ll}
\widehat{F}_{1}(n, t) & \widehat{E}_{1} \hat{R} \\
\widehat{E}_{2} \hat{R}^{\dagger} & \widehat{\bar{F}}_{2}(n, t)
\end{array}\right],
$$

where

$$
e^{-i \hat{N} t}=\left[\begin{array}{ll}
e^{-i\left(K_{z}+\frac{1}{2}\right) t} & 0 \\
0 & e^{-i\left(K_{z}-\frac{1}{2}\right) t}
\end{array}\right]
$$

and

$$
\begin{aligned}
& \widehat{F}_{1}(m, t)=\cos \left(\mu_{1} t\right)-\frac{i \Delta \sin \left(\mu_{1} t\right)}{\mu_{1}}, \quad \widehat{F}_{2}(m, t)=\cos \left(\mu_{2} t\right)+\frac{i \Delta \sin \left(\mu_{2} t\right)}{\mu_{2}}, \\
& \widehat{E}_{j}\left(\mu_{j}, t\right)=-i \lambda \frac{\sin \left(\mu_{j} t\right)}{\mu_{j}}, j=1,2, \quad \mu_{1}^{2}(m)=\frac{\Delta^{2}}{4}+\lambda^{2} \hat{R} \hat{R}^{\dagger}, \mu_{2}^{2}(m)=\frac{\Delta^{2}}{4}+\lambda^{2} \hat{R}^{\dagger} \hat{R}
\end{aligned}
$$

Now let us consider the state $|\theta, \phi\rangle$ which acquires both excited state $|e\rangle$ and ground state $|g\rangle$ for the two-level atom in the following form

$$
|\theta, \phi\rangle=\cos (\theta / 2)|e\rangle+\sin (\theta / 2) \exp (-i \phi)|g\rangle
$$

where $\phi$ is the relative phase of the two atomic levels. To obtain the excited state we have to take $\theta \rightarrow 0$ while to make the wave function describe the particle in the ground state we have to let $\theta \rightarrow \pi$. Assuming that at time $t=0$ the wave function is given by $|\psi(0)\rangle=|\theta, \phi\rangle \otimes|\alpha, k\rangle_{B G}$, where while the $\mathrm{SU}(1,1)$-cavity field is initially in the Barut-Girardello $\mathrm{Su}(1,1)$ coherent states which defined as the eigenstate of the lowering operator $K_{-} ; K_{-}|\alpha, k\rangle_{B G}=\alpha|\alpha, k\rangle_{B G}$

$$
|\alpha, k\rangle_{B G}=\sqrt{\frac{|\alpha|^{2 k-1}}{I_{2 k-1}(2|\alpha|)}} \sum_{m=0}^{\infty} \frac{\alpha^{n}}{\sqrt{\alpha ! \Gamma(2 m+k)}}|m, k\rangle,
$$


where $I_{\zeta}(\chi)=\left(\frac{\chi}{2}\right)^{\zeta} \sum_{m=0}^{\infty} \frac{\left(\frac{\chi}{2}\right)^{2 m}}{m ! \Gamma(m+k+1)}$ is the $\zeta^{t h}$-order modified Bessel function. The wave function of the system is given by $|\psi(t)\rangle=\left(\begin{array}{c}|\psi(t)\rangle_{e} \\ |\psi(t)\rangle_{g}\end{array}\right)|\alpha, k\rangle_{B G}$ where

$$
\begin{aligned}
|\psi(t)\rangle_{e} & =\widehat{F}_{1}(m, t) \cos (\theta / 2)-i \lambda \frac{\sin \left(\mu_{j} t\right)}{\mu_{j}} \hat{R} \sin (\theta / 2) \exp (-i \phi) \\
|\psi(t)\rangle_{g} & =\widehat{F}_{2}(m, t) \sin (\theta / 2) \exp (-i \phi)-i \lambda \frac{\sin \left(\mu_{j} t\right)}{\mu_{j}} \hat{R}^{\dagger} \cos (\theta / 2)
\end{aligned}
$$

Now, one can obtain the reduced atomic density operator via tracing out the field subsystem, where $\rho_{\text {atomic }}=\operatorname{Tr}_{\text {field }}[|\psi(t)\rangle\langle\psi(t)|]$. Then,

$$
\rho_{\text {atom }}(t)=\left(\begin{array}{cc}
|\psi(t)\rangle_{e e}\langle\psi(t)| & |\psi(t)\rangle_{e g}\langle\psi(t)| \\
|\psi(t)\rangle_{g e}\langle\psi(t)| & |\psi(t)\rangle_{g g}\langle\psi(t)
\end{array}\right)
$$

In the following section, we employ the reduced density operator (6) to discuss some quantum information measures for the atomic system.

\section{Information Measurements:}

In this section, we briefly introduce the mathematical definitions of the skew information as an indicator of decoherence and estimator of the system parameters, Tomographic entropy, and relative entropy of quantum coherence.

\subsection{Skew Information}
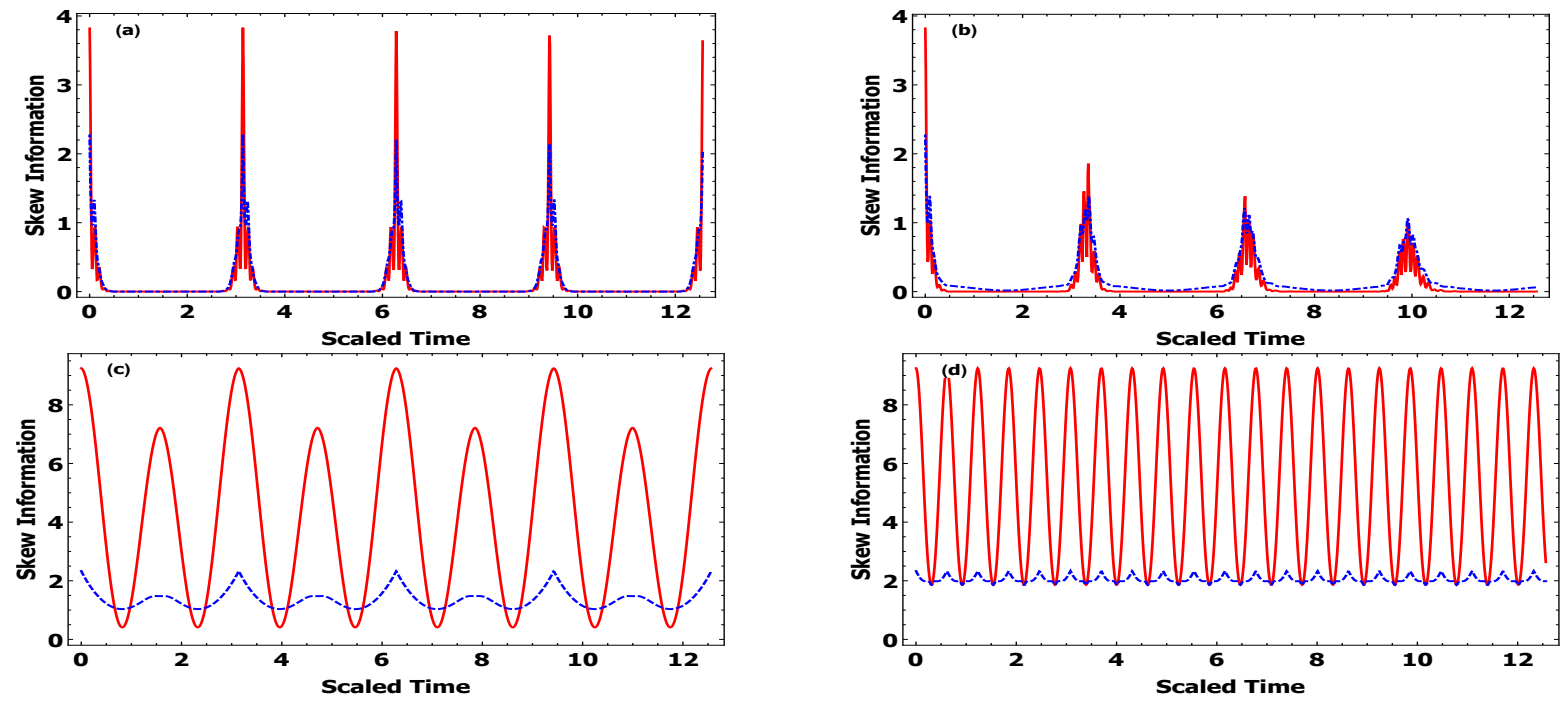

Figure 1: The skew information of $\theta$ parameter if $f\left(k_{z}\right)=1$, with $\phi=\pi / 2, k=0.5, \beta=15$, while $\theta=0$ (red-solid curve) or $\theta=\pi / 2$ (blue-dash curve), (a) $\Delta=0$, (b) $\Delta=10$. (c,d) the same as (a,b) but $f\left(k_{z}\right)=\frac{1}{\sqrt{(m+1)(m+2 k)}}$.

Based on the paradigmatic version of Fisher information [46], which estimates the amount of information of unknown parameters in the quantum states as precisely as possible, is the so-called skew 
information [47].

$$
\mathcal{I}(\rho, \mathcal{K})=-\frac{1}{2} \operatorname{Tr}[\sqrt{\rho}, \mathcal{K}]
$$

where, $\mathcal{K}$ is a fixed conserved observable. For a $2 \times 2$ mixed state, the skew information in terms of the Bloch representation is given by [48],

$$
\mathcal{I}_{\theta}=\frac{2\left|\partial_{\theta} \vec{\tau}\right|^{2}-\left(\vec{\tau} \cdot \partial_{\theta} \vec{\tau}\right)^{2}}{1+\sqrt{1-|\vec{\tau}|^{2}}}+\frac{\left(\vec{\tau} \cdot \partial_{\theta} \vec{\tau}\right)^{2}}{1-|\vec{\tau}|^{2}}
$$

where the polar angle $\theta$ is to be estimated, while $\vec{\tau}=\left\{\tau_{x}, \tau_{y}, \tau_{z}\right\}$ is the real Bloch vector.

The numerical calculations in Fig.(1) show the optimal behaviour of skew information with respect to the polar angle $\theta$. Firstly, we assumed that the perfect cavity of $S U(1,1)$, namely $f\left(k_{z}\right)=1$, and the initial atomic system prepared either in an excited state (red-solid curve) or superposition state (blue-dash curve). The azimuthal angle $\phi=\pi / 2$, the Bargmann index $k=0.5$, and the intensity of Barutâ€"Girardello coherent state $\beta=15$ are set to be fixed for all figures. As it is displayed from Figs.(1.a,b) the non-resonance case plays a key role in the dissipation of the maximum bounds of the skew information, where the maximum bounds of the skew information in resonance case with are $\Delta=0$ grater than that displayed for the non-resonance case with $\Delta=10$. Therefore, the estimation degree of parameter $\theta$ decreases as $\Delta$ increases along the scaled time, hence the decoherence degree increases. The maximum bounds is larger at the scaled time $n \pi \lambda t$, which means that the decoherence has large values. The influence of the nonlinear cavity on the skew information is displayed in Figs.(1). c,d), where

we set $f\left(k_{z}\right)=\frac{1}{\sqrt{(m+1)(m+2 k)}}$. The optimal behaviour of $\mathcal{I}_{\theta}$ shows that the detuning parameter has a clear effect on the number of oscillations, where the oscillations between the upper and lower values increases as the detuning increases. However, the lower bounds at the non-resonance case $(\Delta=10)$ are higher than those displayed in the resonance case. This means that, the decoherence degree is very large comparing with those shown in Figs.(1,a,b). Moreover, the maximum and minimum bounds depend on the initial atomic states, where the maximum bounds that are depicted in the red-solid curve as the atomic system is prepared in an excited state are much larger than those displayed in the blue-dash curve.

\subsection{Tomographic Entropy:}

The tomographic entropy is introduced to illustrate the entanglement of the spin states of the quantum system [49]. For a generic $2 \times 2$ density matrix, the tomographic entropy is defined as [15],

$$
\mathcal{T}=\frac{-1}{\sqrt{2 \pi}} \int_{0}^{2 \pi} \int_{0}^{\pi} \sum_{i=1}^{2} Q_{i}(\Theta, \Phi) \ln Q_{i}(\Theta, \Phi) \sin \Theta d \Theta d \Phi
$$

here, $Q_{i}(\Theta, \Phi)$ are the generic spin tomograms, with,

$$
\begin{aligned}
& Q_{1}(\Theta, \Phi)=\rho_{11} \cos ^{2} \Theta / 2+\rho_{22} \sin ^{2} \Theta / 2+\sin \Theta \Re\left[\rho_{12} e^{i \Phi}\right] \\
& Q_{2}(\Theta, \Phi)=\rho_{22} \cos ^{2} \Theta / 2+\rho_{11} \sin ^{2} \Theta / 2-\sin \Theta \Re\left[\rho_{12} e^{i \Phi}\right]
\end{aligned}
$$

In Fig.(2), we investigate the quantum entanglement of the ideal/defect $\mathrm{SU}(1,1)$ cavity field via the tomographic entropy, where the same conditions of the previous case in Fig.(1) are considered. Fig.(2.a) describes the optimal behaviour of the tomographic entropy in the resonance case, where it ordinary osculates between $2.5 \mapsto 3.5$. It is seen that the function $\mathcal{T}$ tends to zero periodically with scaled time 

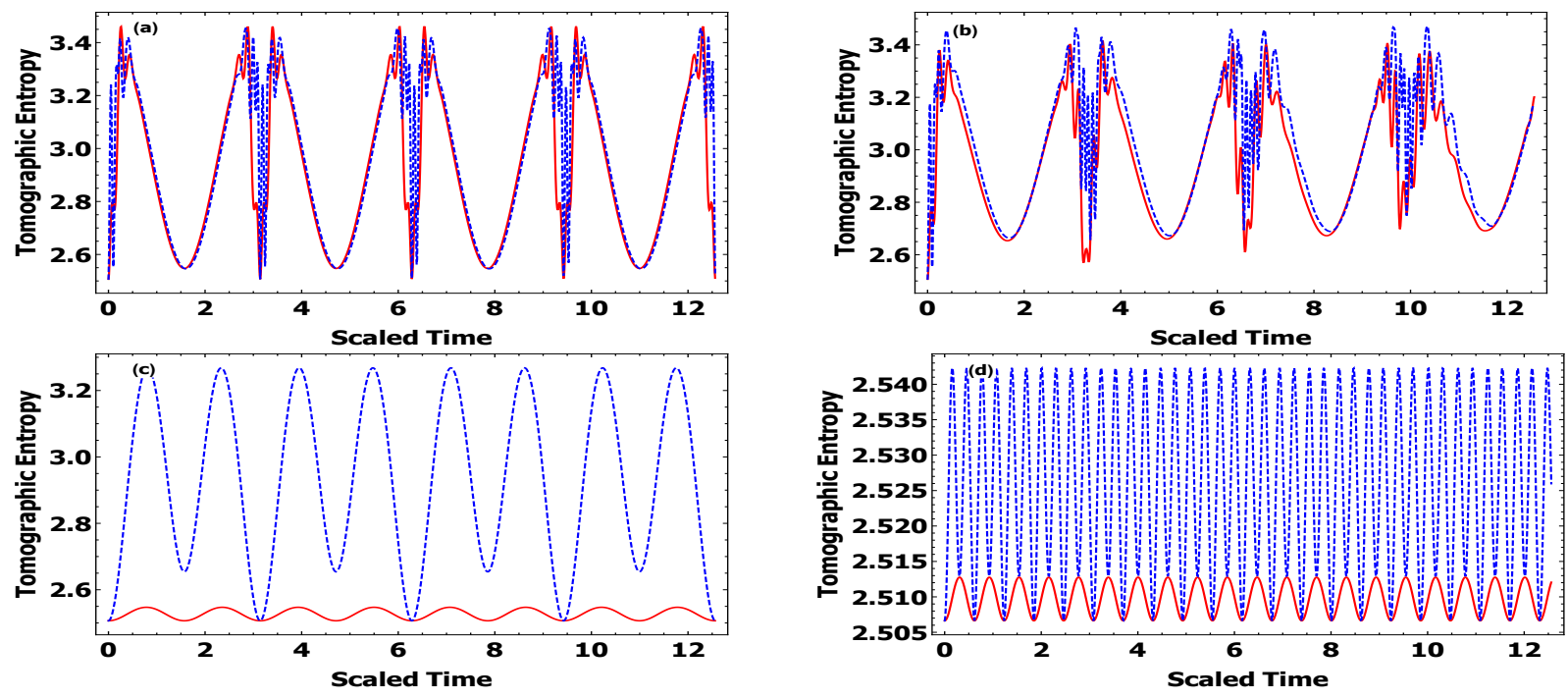

Figure 2: Tomographic entropy with the same as Fig.(1)

$\lambda t=n \pi$, which means, the system returns to the separable state. The degree of entanglement for excited and superposition states are extremely identical between the time intervals $\lambda t=n \pi$. Meanwhile. the effect of the non-resonance case is disclosed in fig. (2,b), where the lower bounds of the tomographic entropy decrease as the scaled time increases. That means the separable state returned to a partially entangled state, while the upper bounds are fixed. On the other hand, the non-linear cavity has a remarkable influence, where the large detuning increases the numbers of osculations and decreases the upper bounds of the function $\mathcal{T}$. Consequently, the entanglement decreases as the detuning increases and the entangled states approach separable states. Interestingly, there is an eminent correlation between the skew information and the sign of entanglement, where the weakening of entanglement corresponding to the strengthening and growth of the skew information.

\subsection{Relative Entropy of Coherence:}

According to relative von Neumann entropy, Baumgratz and et.al. [50] are employed the relative entropy to quantify the quantum coherence. The relative entropy of coherence was defined as a minimum relative entropy between the set of all incoherent states $I$ and the given state $\rho$,

$$
\mathcal{C}=\min _{\epsilon \in I} \mathcal{S}(\rho \| \epsilon)=\operatorname{Tr}[\rho \ln \rho]-\operatorname{Tr}\left[\rho_{\text {diag }} \ln \rho_{\text {diag }}\right]
$$

where, $I$ is the set of all incoherent states. $\rho_{\text {diag }}=\sum_{i} \rho_{i i}|i\rangle\langle i|$ is the diagonal element of $\rho$.

Finally, we discuss the influence of the initial atomic system $\theta$ and different resonances causes $\Delta$ on the behaviour of the relative coherence, with the same conditions in Fig.(1). Fig.(3) a logical correlation between the tomographic entropy and quantum coherence, this correlation is correct because of any entangled state subset of coherence states. The initial settings of the atomic state play a central role in the coherence degree. The non-resonance case and non-linear cavity improve the coherence degree for the initial superposition state. Also, the non-resonance case and linear cavity SU $(1,1)$ reduce the coherence degree as time developed. 

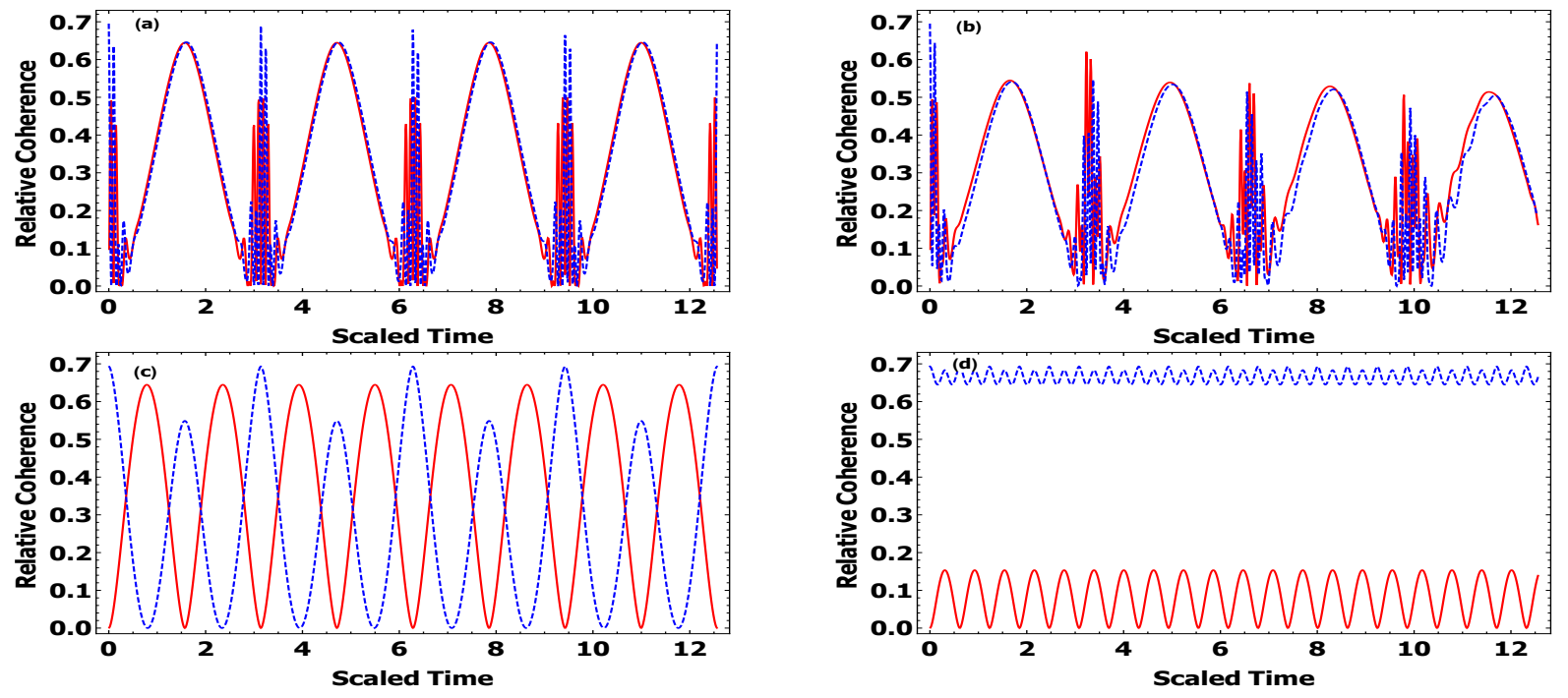

Figure 3: Relative entropy of coherence with the same as Fig.(1)

\section{Conclusion:}

A system consists of a single two-level atom in $S U(2)$ interacts locally with linear/ non-linear $S U(1,1)$ quantum systems. It is considered that the initial atomic state is either prepared in its excited state or a superposition state. We discussed the influence of linear/ non-linear quantum system, which is represented by ideal/ imperfect field operators, on the behavior of three quantum quantities. These quantities include the skew information, the tomographic entropy, and the relative entropy of coherence. The phenomena of the collapse and revival behavior of the three functions are depicted clearly when the atomic state is initially prepared in the excited state. However, if the system is initially prepared in a superposition state, the predicted behavior of the three functions is much larger than that displayed for the excited state.

The influence of a non-linear state on the optimal behavior of the estimation degree, the entanglement, and coherence in the initial excited/ superposition state are discussed. The results are shown that the detuning parameter is used as a control parameter, that maximizes/ minimize the three phenomena. This means that by controlling the detuning, one can increase the ability to suppress the separability induced by the imperfect of quantum system. However, the oscillation's number increases as the detuning increases and consequently the lower/upper bounds of the three phenomena are improved. Similar effect is predicted where the cavity is prepared in ideal $S U(1,1)$. The maximum/ minimum bounds of the three quantifiers are displayed at large detuning parameter.

\section{References}

[1] Laszlo Gyongyosi and Sandor Imre. Entanglement access control for the quantum internet. Quantum Inf. Process., 18(4):1-17, 2019.

[2] Yinghua Ji and Juju Hu. Control of quantum entanglement and entropic uncertainty in open quantum system: Via adjusting ohmic parameter. Physica E, 114:113583, 2019.

[3] Dongyang Wang, Yong Liu, Jiangfang Ding, Xiaogang Qiang, Yingwen Liu, Anqi Huang, Xiang Fu, Ping Xu, Mingtang Deng, Xuejun Yang, et al. Remote-controlled quantum computing by quantum entanglement. Opt. Lett., 45(22):6298-6301, 2020. 
[4] N. Metwally. Enhancing entanglement, local and non-local information of accelerated two-qubit and two-qutrit systems via weak-reverse measurements. EPL, 116(6):60006, 2017.

[5] Xuexiang $\mathrm{Xu}$, Liyun $\mathrm{Hu}$, and Zeyang Liao. Improvement of entanglement via quantum scissors. JOSA B, 35(1):174-181, 2018.

[6] MY Abd-Rabbou, N Metwally, MMA Ahmed, and A-SF Obada. Suppressing the information losses of accelerated qubit-qutrit system. Int. J. Quantum Inf., 17(04):1950032, 2019.

[7] Min Yu, Huan Zhang, Wei Ye, Kuizheng Zhang, Ying Xia, and Liyun Hu. Improvement of entanglement via catalytic quantum scissors. Optik, 241:167252, 2021.

[8] Johann von Neumann. Mathematische grundlagen der quantenmechanik springer, 1932.

[9] Simon JD Phoenix and PL Knight. Establishment of an entangled atom-field state in the JaynesCummings model. Phys. Rev. A, 44(9):6023, 1991.

[10] Heng Fan, Keiji Matsumoto, and Hiroshi Imai. Quantify entanglement by concurrence hierarchy. J. Phys. A, 36(14):4151, 2003.

[11] Ryszard Horodecki, Paweł Horodecki, Michał Horodecki, and Karol Horodecki. Quantum entanglement. Rev. Mod. Phys., 81:865-942, Jun 2009.

[12] You Zhou, Pei Zeng, and Zhenhuan Liu. Single-copies estimation of entanglement negativity. Phys. Rev. Lett., 125:200502, Nov 2020.

[13] William K. Wootters. Entanglement of formation of an arbitrary state of two qubits. Phys. Rev. Lett., 80:2245-2248, Mar 1998.

[14] M Sebawe Abdalla and M.M.A. Ahmed. Entropy squeezing and entanglement of the interaction between SU (1, 1) and SU (2) quantum systems. Opt. Commun., 284(7):1933-1940, 2011.

[15] Vladimir N Chernega, Olga V Man'ko, Vladimir I Man'ko, Oleg V Pilyavets, and Vadim G Zborovskii. Tomographic characteristics of spin states. J. Russ. Laser Res., 27(2):132-166, 2006.

[16] Abdullah M Almarashi, Ali Algarni, S Abdel-Khalek, GA Abd-Elmougod, and Mohammad Z Raqab. Quantum extropy and statistical properties of the radiation field for photonic binomial and even binomial distributions. J Russ. Laser Res., 41(4):334-343, 2020.

[17] M.Y. Abd-Rabbou, N. Metwally, M.M.A. Ahmed, and A.-S.F. Obada. Wigner function of noisy accelerated two-qubit system. Quantum Inf. Process., 18(12):1-19, 2019.

[18] A.-B.A. Mohamed, E.M. Khalil, N. Metwally, and H. Eleuch. Local two-atom correlations induced by a two-mode cavity under nonlinear media: Quantum uncertainty and quantum Fisher information. Results Phys., page 104975, 2021.

[19] Edwin T Jaynes and Frederick W Cummings. Comparison of quantum and semiclassical radiation theories with application to the beam maser. Proceedings of the IEEE, 51(1):89-109, 1963.

[20] M. Sebawe Abdalla, M.M.A. Ahmed, and A.-S.F. Obada. Dynamics of a non-linear JaynesCummings model. Physica A: Statistical Mechanics and Its Applications, 162(2):215-240, 1990.

[21] Bruce W Shore and Peter L Knight. The jaynes-cummings model. J. Mod. Opt., 40(7):1195-1238, 1993. 
[22] G Sadiek, EI Lashin, and M Sebawe Abdalla. Entanglement of a two-qubit system with anisotropic XYZ exchange coupling in a nonuniform time-dependent external magnetic field. Physica B, 404(1213):1719-1728, 2009.

[23] D.A.M. Abo-Kahla, M.Y. Abd-Rabbou, and N Metwally. The orthogonality speed of two-qubit state interacts locally with spin chain in the presence of Dzyaloshinsky-Moriya interaction. Laser Phys. Let., 18(4):045203, 2021.

[24] F Jahanbakhsh and MK Tavassoly. The field-field and dipole-dipole coupling effects on the entanglement of the interaction between two qutrits with a two-mode field. Mod. Phys. Let. A, 35(22):2050183, 2020.

[25] Ling-Juan Feng, Yu You, Heng-Xing Dong, Feng-Chao Wang, and Shang-Qing Gong. Enhancing cross-Kerr coupling via mechanical parametric amplification. Optics Express, 29(18):28835-28842, 2021.

[26] M Sebawe Abdalla, Jan Peřina, and Jaromír Křepelka. Statistical properties of multiphoton timedependent three-boson coupled oscillators. JOSA B, 23(6):1146-1160, 2006.

[27] M. Sebawe Abdalla, M.M.A. Ahmed, E.M. Khalil, and A.-S.F. Obada. The interaction between a single two-level atom coupled to an N-level quantum system through three couplings. Ann. Phys., 364:168-181, 2016.

[28] Eman MA Hilal, S Alkhateeb, S Abdel-Khalek, EM Khalil, and Amjaad A Almowalled. Quantum scheme for N-level atom interacting with a two two-level atom: Atomic Fisher information and entropy squeezing. Alexandria Engineering Journal, 59(3):1259-1264, 2020.

[29] Abdallah A Nahla, M. M. A. Ahmed, and Sultan Z Alamri. Analytical computation of nonclassical behavior for asymmetric two two-level atoms interacting with $\mathrm{SU}(1,1)$ quantum system. Europ. Phys. J. D, 73(3), 2019.

[30] Abdel-Baset A Mohamed, Mohamed Sebawe Abdalla, and A.-S. F. Obada. Quantum effects due to the interaction between $\mathrm{SU}(1,1)$ and $\mathrm{SU}(2)$ quantum systems with damping. Europ. Phys. J. D, 71(9):1-8, 2017.

[31] M Sebawe Abdalla, E.M. Khalil, A.S.-F. Obada, J Peřina, and J Křepelka. Linear entropy and squeezing of the interaction between two quantum system described by SU $(1,1)$ and SU (2) lie group in presence of two external terms. AIP Advances, 7(1):015013, 2017.

[32] A.-S.F. Obada, Nawal A Alshehri, E.M Khalil, S Abdel-Khalek, and HF Habeba. Entropy squeezing and atomic Wehrl density for the interaction between $\mathrm{SU}(1,1)$ lie algebra and a three-level atom in presence of laser field. Results Phys., 30:104759, 2021.

[33] Ronald Aylmer Fisher. Theory of statistical estimation. In Mathematical proceedings of the Cambridge philosophical society, volume 22, pages 700-725. Cambridge University Press, 1925.

[34] N Metwally. Fisher information of a single qubit interacts with a spin-qubit in the presence of a magnetic field. Physica E, 100:14-23, 2018.

[35] S Abdel-Khalek, EM Khalil, A-BA Mohamed, M Abdel-Aty, and HR Besbes. Response of quantum Fisher information, variance entropy squeezing and entanglement to the intrinsic decoherence of two non-degenerate fields interacting with two qubits. Alexandria Engineering Journal, 59(6):51475154, 2020. 
[36] H Fakhri and A Dehghani. Coherency of SU (1, 1)-Barut-Girardello type and entanglement for spherical harmonics. J. math. phys., 50(5):052104, 2009.

[37] SR Miry and M.K. Tavassoly. Generation of a class of SU $(1,1)$ coherent states of the GilmorePerelomov type and a class of SU (2) coherent states and their superposition. Physica Scripta, 85(3):035404, 2012.

[38] A-BA Mohamed and N Metwally. Non-classical correlations based on skew information for an entangled two qubit-system with non-mutual interaction under intrinsic decoherence. Ann. Phys., 381:137-150, 2017.

[39] Yi-Hao Sheng, Jian Zhang, Yuan-Hong Tao, and Shao-Ming Fei. Applications of quantum coherence via skew information under mutually unbiased bases. Quantum Inf. Process., 20(2):1-12, 2021.

[40] A-BA Mohamed, EM Khalil, and M.Y. Abd-Rabbou. Quantum effects induced by two classical fields in a coherent cavity field containing two two-level atoms. Physica E, page 114839, 2021.

[41] T Holstein and Hl Primakoff. Field dependence of the intrinsic domain magnetization of a ferromagnet. Physical Review, 58(12):1098, 1940.

[42] Lawrence R Mead and N Papanicolaou. Holstein-primakoff theory for many-body systems. Phys. Rev. B, 28(3):1633, 1983.

[43] Stig Stenholm. A bargmann representation solution of the Jaynes - Cummings model. Opt. Commun., 36(1):75-78, 1981.

[44] Stig Stenholm. Quantum theory of electromagnetic fields interacting with atoms and molecules. Phys. Rep., 6(1):1-121, 1973.

[45] Stig Stenholm. Saturation effects in rf spectroscopy. iii. mixing of nearly degenerate levels by strong off-resonant fields. Journal of Physics B: Atomic and Molecular Physics, 6(6):1097, 1973.

[46] Shunlong Luo. Wigner-Yanase skew information and uncertainty relations. Phys. Rev. Lett., 91:180403, Oct 2003.

[47] Eugene P Wigner and Mutsuo M Yanase. Information contents of distributions. In Part I: Particles and Fields. Part II: Foundations of Quantum Mechanics, pages 452-460. Springer, 1997.

[48] Wei Zhong, Zhe Sun, Jian Ma, Xiaoguang Wang, and Franco Nori. Fisher information under decoherence in Bloch representation. Phys. Rev. A, 87:022337, Feb 2013.

[49] VV Dodonov and VI Man'ko. Positive distribution description for spin states. Phys. Let. A, 229(6):335-339, 1997.

[50] T. Baumgratz, M. Cramer, and M. B. Plenio. Quantifying coherence. Phys. Rev. Lett., 113:140401, Sep 2014. 